Vietnam Journal of Mechanics, VAST, Vol. 34, No. 1 (2012), pp. $1-6$

\title{
A NEW VIEW OF THE BUBNOV-GALERKIN METHOD IN THE LINEARIZATION CONTEXT
}

\author{
N. D. Anh ${ }^{1}$ and I. Elishakoff ${ }^{2}$ \\ ${ }^{1}$ Institute of Mechanics, Hanoi, Vietnam \\ ${ }^{2}$ Florida Atlantic University, Boca Raton, USA
}

\begin{abstract}
In the study an extension of the Bubnov-Galerkin method in terms of the equivalent linearization method is presented. It is combined with sequential linearization and nonlinear procedure to yield a new method for solving nonlinear equations which can improve the accuracy when the nonlinearity is strong. For illustration the Duffing oscillator is considered to show the effectiveness of the proposed method.
\end{abstract}

Keywords: Bubnov-Galerkin, nonlinear, stochastic, Duffing.

\section{INTRODUCTION}

Bubnov-Galerkin method is one of most popular approximate methods in many fields of applied mechanics since the method is general in scope and can be used for both conservative and nonconservative, both linear and nonlinear systems. The idea was apparently first suggested in 1913 by Bubnov [1], whereas the first paper along with elaborative examples was written in 1915 by Galerkin [2]. In 1937 Duncan [3] published the first comprehensive review of the method in the Western literature. For a given differential equation the Bubnov-Galerkin method approximates the sought solution as a linear combination of comparison functions and requires the orthogonality of the residual to each of comparison functions. In this context the Bubnov-Galerkin method is also known as a weighted residual method [4]. Although the method can be used for both linear and nonlinear systems, it is known that the accuracy of the method decreases when the nonlinearity becomes larger. Elishakoff [5] connected the Bubnov-Galerkin method with the equivalent linearization method.

In this paper a representation of the Bubnov-Galerkin method in terms of the equivalent linearization method is presented and a dual approach is subsequently adopted to suggest a new method for solving nonlinear equations. This combined approach allows improving the accuracy when the nonlinearity is strong. For illustration the Duffing oscillator and a nonlinear vibration of string are considered to demonstrate the effectiveness of the proposed method. 


\section{VIEW OF BUBNOV-GALERKIN METHOD FROM THE STOCHASTIC LINEARIZATION CONTEXT}

Consider the following nonlinear differential equation

$$
L(W)=0
$$

where $L$ is a differential operator, $W$ is an unknown function which satisfies specific boundary conditions. We replace approximately the function $L(W)$ by a linear term

$$
L(W) \approx k W
$$

where the coefficient $k$ is chosen from the condition of minimum mean square deviation criterion

$$
\left\langle(L(W)-k W)^{2}\right\rangle \rightarrow \min _{k}
$$

where $\langle$.$\rangle is a functional which is usually taken in a form of integration operator over$ the domain of the operator $L(W)$. One gets from (3):

$$
k=\frac{<L(W) W>}{<W^{2}>} .
$$

Using Eq. (2) one obviously observes that Eq. (1) is satisfied approximately if

$$
k=0 \text {. }
$$

Alternatively, from Eq. (4) we get another condition

$$
<L(W) W>=0 \text {. }
$$

The Eq. (6) is known as the orthogonality of the residual $L(W)$ to the comparison function $W$. Hence, the Bubnov-Galerkin method can be employed in terms of the equivalent linearization method. Moreover, the accuracy of the method can be expected to be improved by using the dual approach developed recently by Anh [5]. Suppose that the operator $L(W)$ can be expressed as a sum of two operators:

$$
L(W)=M(W)+N(W)
$$

where $M$ is the linear operator and $N$ is the nonlinear operator.

At this juncture sequential linearization and nonlinearization approach will be applied to the nonlinear operator $N(W)$. Note that Anh [5] refers to it as the dual approach. By the first step we replace $N(W)$ approximately by a linear term

$$
N(W) \approx \alpha W .
$$

The difference can be measured by the following expression:

$$
\left\langle[N(W)-\alpha W]^{2}\right\rangle \text {. }
$$

Second step consists in the replacement of the equivalent linear term $\alpha W$ by the original nonlinear term $N(W)$ but now multiplied by a factor. The difference can be measured by the following expression:

$$
\left\langle[\alpha W-\beta N(W)]^{2}\right\rangle .
$$


These two procedures should be optimized together, as follows,

$$
D \equiv\left\langle[N(W)-\alpha W]^{2}\right\rangle+\left\langle[\alpha W-\beta N(W)]^{2}\right\rangle \rightarrow \min _{\alpha, \beta}
$$

The coefficients $\alpha, \beta$ are determined from the following requirements

$$
\frac{\partial(D)}{\partial \alpha}=0, \quad \frac{\partial(D)}{\partial \beta}=0
$$

which yield

$$
\begin{gathered}
\alpha=\frac{1}{2-\gamma} \frac{\langle N(W) W\rangle}{\left\langle W^{2}\right\rangle} \\
\beta=\frac{\eta}{2-\gamma}
\end{gathered}
$$

where

$$
\gamma=\frac{\langle N(W) W\rangle^{2}}{\left\langle(N(W))^{2}\right\rangle\left\langle W^{2}\right\rangle}
$$

To reiterate, using Eqs. (7) and (8), the original equation (1) is replaced approximately by the following one:

$$
M(W)+\alpha W=0
$$

Applying to Eq. (14) the classical equivalent linearization method or the BubnovGalerkin method one gets

$$
\langle M(W) W\rangle+\alpha\left\langle W^{2}\right\rangle=0 .
$$

Substituting Eqs. (11) and (13) into Eq. (15) yields

$$
\langle M(W) W\rangle+\frac{1}{2-\gamma}\langle N(W) W\rangle=0
$$

or, noting Eq. (7), one gets

$$
\langle L(W) W\rangle+\frac{\gamma-1}{2-\gamma}\langle N(W) W\rangle=0 .
$$

Substituting (13) into (17) results in the following equation

$$
\langle L(W) W\rangle+\frac{\langle N(W) W\rangle^{2}-\left\langle(N(W))^{2}\right\rangle\left\langle W^{2}\right\rangle}{2\left\langle(N(W))^{2}\right\rangle\left\langle W^{2}\right\rangle-\langle N(W) W\rangle^{2}}=0 .
$$

It is seen that the equation (18) differs from the Bubnov-Galerkin equation (6). Moreover, in general the orthogonality of the residual $L(W)$ to the comparison function $W$ is not required anymore. Because in addition to the first term that coincides with the left side of Eq. (6), Eq. (18) contains an additional term. 


\section{ILLUSTRATIVE EXAMPLE: DUFFING OSCILLATOR}

For illustration of the effectiveness of the equation (18) we consider the well known Duffing oscillator under random excitation

$$
L(W) \equiv \frac{d^{2} W}{d t^{2}}+2 h \frac{d W}{d t}+\omega_{0}^{2} W+\mu W^{3}-\sigma \dot{\xi}(t)=0
$$

where $\dot{\xi}(t)$ is the white noise process with unit intensity. The comparison function $W$ is taken as a solution of the corresponding linear equation:

$$
\frac{d^{2} W}{d t^{2}}+2 h \frac{d W}{d t}+\omega_{0}^{2} W+k W-\sigma \dot{\xi}(t)=0 .
$$

Substituting (20) into (19) gives the residual

$$
L(W)=\mu W^{3}-k W .
$$

Thus by using the Bubnov-Galerkin method one gets

$$
\langle L(W) W\rangle=\left\langle\left(\mu W^{3}-k W\right) W\right\rangle=0
$$

or

$$
k=\mu \frac{\left\langle W^{4}\right\rangle}{\left\langle W^{2}\right\rangle}=3 \mu\left\langle W^{2}\right\rangle
$$

where $<$. $>$ is the mathematical expectation operator. The system of two equations (20) and (23) allows obtaining the unknown $<W^{2}>$.

Using the present method one considers $L(W)$ as a sum of two operators $M(W)$ and $N(W)$ where

$$
M(W)=\frac{d^{2} W}{d t^{2}}+2 h \frac{d W}{d t}+\omega_{0}^{2} W-\sigma \dot{\xi}(t), \quad N(W)=\mu W^{3} .
$$

Substituting Eqs. (21), (23) into (18) gives

$$
\left\langle\left(\mu W^{3}-k W\right) W\right\rangle+\frac{\mu^{2}\left\langle W^{4}\right\rangle^{2}-\mu^{2}\left\langle W^{6}\right\rangle\left\langle W^{2}\right\rangle}{2 \mu^{2}\left\langle W^{6}\right\rangle\left\langle W^{2}\right\rangle-\mu^{2}\left\langle W^{4}\right\rangle^{2}} \mu\left\langle W^{4}\right\rangle=0 .
$$

Noting that

$$
\left\langle W^{2 n}\right\rangle=1 \cdot 3 \cdot 5 \cdots(2 n-1)\left\langle W^{2}\right\rangle^{n}
$$

one gets

$$
3 \mu\left\langle W^{2}\right\rangle^{2}-k\left\langle W^{2}\right\rangle+\frac{(15-9)\left\langle W^{2}\right\rangle^{4}}{(30-9)\left\langle W^{2}\right\rangle^{4}} 3 \mu\left\langle W^{2}\right\rangle^{2}=0
$$

or

$$
k=\frac{15}{7} \mu\left\langle W^{2}\right\rangle .
$$

It is remarkable that Eqs. (23) and (28) differ from each other.

The results of mean-square response of Duffing oscillator (19) obtained by the Bubnov-Galerkin method $\left(<W^{2}>_{\mathrm{GM}}\right)$ and present method $\left(<W^{2}>_{\mathrm{PM}}\right)$ are compared with the exact ones $\left(<W^{2}>_{\mathrm{E}}\right)$ in Table 1 . It is seen that the errors of mean-square responses determined by the present method are much less than the errors of mean-square 
response obtained by the straightforward Bubnov-Galerkin method for the case of strong nonlinearity.

Table 1. Mean-Square Responses $<W^{2}>$ of Duffing oscillator for $\sigma^{2} /(4 h)=$ $1, \omega_{0}=1$

\begin{tabular}{|c|c|c|c|c|c|}
\hline$\gamma$ & $\left(<W^{2}>_{\mathrm{E}}\right)$ & $\left(<W^{2}>_{\mathrm{GM}}\right)$ & error $\%$ & $\left(<W^{2}>_{\mathrm{PM}}\right)$ & error \% \\
\hline 0.1 & 0.8176 & 0.8054 & 1.4876 & 0.8465 & 3.5352 \\
\hline 0.5 & 0.5792 & 0.5486 & 5.2861 & 0.6062 & 4.6670 \\
\hline 1.0 & 0.4679 & 0.4343 & 7.1938 & 0.4885 & 4.4082 \\
\hline 5.0 & 0.2543 & 0.2270 & 10.7384 & 0.2624 & 3.1708 \\
\hline 10 & 0.1889 & 0.1667 & 11.7721 & 2.6697 & 2.6697 \\
\hline 50 & 0.0904 & 0.0784 & 13.2721 & 1.8539 & 1.8539 \\
\hline 100 & 0.0650 & 0.0561 & 13.6491 & 0.0660 & 1.6331 \\
\hline
\end{tabular}

It should be noted that the present method can also be used for investigating deterministic nonlinear vibrations. In fact, consider a particular case of Eq. (19) where $h, \sigma=0$, i.e. we have free periodic vibrations described by the following differential equation:

$$
L(W) \equiv \frac{d^{2} W}{d t^{2}}+\omega_{0}^{2} W+\mu W^{3}=0 .
$$

The corresponding linear equation reads

$$
\frac{d^{2} W}{d t^{2}}+\omega_{0}^{2} W+k W=0
$$

where the equivalent linear term is determined by Eq. (25) but the operator $\langle$.$\rangle is taken$ as follows

$$
<\cdot>=\frac{1}{l} \int_{0}^{l}(\cdot) d \varphi
$$

where $l$ is a positive value. For the case $l=2 \pi$ substituting $W=\operatorname{acos} \phi$ into (25) yields

$$
\begin{aligned}
\left\langle\left[\mu(a \cos \varphi)^{3}\right.\right. & -k a \cos \varphi] a \cos \varphi\rangle+ \\
& +\frac{\mu^{2}\left\langle(a \cos \varphi)^{4}\right\rangle^{2}-\mu^{2}\left\langle(a \cos \varphi)^{6}\right\rangle\left\langle(a \cos \varphi)^{2}\right\rangle}{2 \mu^{2}\left\langle(a \cos \varphi)^{6}\right\rangle\left\langle(a \cos \varphi)^{2}\right\rangle-\mu^{2}\left\langle(a \cos \varphi)^{4}\right\rangle^{2}} \mu\left\langle(a \cos \varphi)^{4}\right\rangle=0 .
\end{aligned}
$$

Noting

one gets

$$
\left\langle\cos \varphi^{2}\right\rangle=\frac{1}{2}, \quad\left\langle\cos \varphi^{4}\right\rangle=\frac{3}{8}, \quad\left\langle\cos \varphi^{6}\right\rangle=\frac{5}{16}
$$

$$
k=\frac{15}{22} \mu a^{2} .
$$

Hence, the frequency of the free Duffing oscillator (29) obtained by the present method is

$$
\omega=\left(\omega_{0}^{2}+\frac{15}{22} \mu a^{2}\right)^{1 / 2} .
$$


That approximate frequency differs from the approximate frequency

$$
\omega=\left(\omega_{0}^{2}+\frac{3}{4} \mu a^{2}\right)^{1 / 2}
$$

obtained by the classical linearized method. The accuracy of two approximate frequencies (35), (36) can be investigated by comparing with the exact one [6].

\section{CONCLUSION}

In the study the Bubnov-Galerkin method in the context of the equivalent linearization method is first reiterated. A sequential linearization and nonlinearization method is then adopted to suggest a new method for solving nonlinear equations. This dual or sequential method can improve the accuracy of the equivalent linearization technique when the nonlinearity is strong. For illustration the Duffing oscillator subjected to random excitation is considered to demonstrate the effectiveness of the proposed methodology. The method appears to have a certain potential, it ought to be explored for wider nonlinear classes.

\section{ACKNOWLEDGEMENTS}

The research reported in this paper is supported by Vietnam National Foundation for Science and Technology Development, and the Department of Ocean and Mechanical Engineering, Florida Atlantic University.

\section{REFERENCES}

[1] Bubnov I. G., Reviews of Professor Kirpichev, Belzetskii, Bubnov and Kolosoff on Works of Professor Timoshenko, awarded the D. I. Zhuravskii Prize, Sbornik St. Peterburgskogo Instituta Inzhenerov Putei Soobchenia (Collection of St. Petersburg Institute of Transportation Engineering), 81 (1913) 1 - 40 (see also Bubnov I. G., 1956, Selected Works, Sudpromgiz Publishers, Leningrad, pp. 136-139, in Russian).

[2] Galerkin B. G., Rods and Plates, Series in Some Questions of Elastic Equilibrium of Rods and Plates, Vestnik Inzhenerov i Technikov, 19 (1915) 897 - 908 (English Translation, in W. P. Rodden, Theoretical and Computational Aeroelasticity, (2011) 700 - 745, Crest Publishing).

[3] Duncan W. J., Galerkin's Method in Mechanics and Differential Equations, Aeronautical Research Committee Reports and Memoranda, (1937) N 1848.

[4] Elishakoff I., Stochastic Linearization Technique: a New Interpretation and a Selective Review, Shock and Vibration Digest, 32 (2000) 179 - 188.

[5] Anh N. D., Dualtiy in the Analysis of Responses to Nonlinear Systems, Vietnam Journal of Mechanics, 32(4) (2010) 263 - 266.

[6] N. N. Bogoliubov, Yu. A. Mitropolski, Asymptotic Methods in the Theory of NonlinearOoscillations, Gordon and Beach, New York, (1961). 